\title{
The next big thing is actually small
}

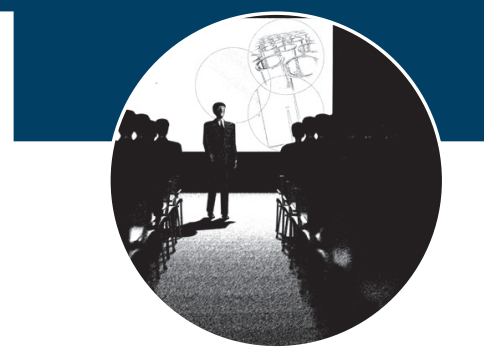

Recent developments in materials, surface modifications, separation schemes, detection systems and associated instrumentation have allowed significant advances in the performance of lab-on-a-chip devices. These devices, also referred to as micro total analysis systems ( $\mu \mathrm{TAS}$ ), offer great versatility, high throughput, short analysis time, low cost and, more importantly, performance that is comparable to standard bench-top instrumentation. To date, $\mu$ TAS have demonstrated advantages in a significant number of fields including biochemical, pharmaceutical, military and environmental. Perhaps most importantly, $\mu$ TAS represent excellent platforms to introduce students to microfabrication and nanotechnology, bridging chemistry with other fields, such as engineering and biology, enabling the integration of various skills and curricular concepts. Considering the advantages of the technology and the potential impact to society, our research program aims to address the need for simpler, more affordable, faster and portable devices to measure biologically active compounds. Specifically, the program is focused on the development and characterization of a series of novel strategies towards the realization of integrated microanalytical devices. One key aspect of our research projects is that the developed analytical strategies must be compatible with each other; therefore, enabling their use in integrated devices. The program combines spectroscopy, surface chemistry, capillary electrophoresis, electrochemical detection and nanomaterials. This article discusses some of the most recent results obtained in two main areas of emphasis: capillary electrophoresis, microchip-capillary electrophoresis, electrochemical detection and interaction of proteins with nanomaterials.

\section{Capillary electrophoresis, microchip- CE \& electrochemical detection}

Since its development in the early 80 s, capillary electrophoresis (CE) has become one of the most powerful and versatile analytical techniques available. CE encompasses a family of separation techniques driven by an electric potential difference applied across a capillary $(<100 \mu \mathrm{m}$ ID) filled with an electrolyte solution. When a potential difference is applied across the capillary, bulk flow of the solution is generated by a process referred to as electro-osmotic flow (EOF) and analytes can be electrophoretically separated based on their charge:size ratio, the magnitude of the applied potential and the solution conditions.

CE offers several advantages when compared with other analytical techniques such as GC and HPLC. First, a wide variety of compounds ranging from small inorganic ions to large organic molecules can be analyzed with the same instrument and even the same capillary by simply adjusting the composition of the electrolyte solution. Second, the flat flow profile generated inside the capillary (due to the EOF) produces very narrow peaks and highly efficient separations. Third, only nanoliters of sample and microliters of buffer are used in each CE analysis, reducing cost and waste. Fourth, the instrumentation required for $\mathrm{CE}$ is relatively simple [1] and does not require the use of mechanical pumps; therefore, enabling the development of miniaturized systems. In this way, $\mathrm{CE}$ and microchip-CE provide high-speed, high-throughput, highly efficient and reliable separations that can be performed at the point of interest.

Many detection systems have been integrated to $\mathrm{CE}$ and microchip-CE devices. Among them, electrochemical detection offers remarkable mass sensitivity (approaching that of fluorescence), inherent miniaturization of both the detector and control instrumentation [1], low cost, minimal power demands, high compatibility with microfabrication technologies and independence from sample turbidity, optical path length or substrate clarity. In addition, electrochemical detection does not scale linearly with decreasing electrode size, a limitation to most optical methods. Therefore, it is easier to miniaturize the detection elements and still maintain a significant signal level. Furthermore, with the use of microfabrication techniques it is possible to integrate multiple electrodes into a single microchip, thus improving the ability to perform more selective and/or more sensitive detection modes. Finally, electrodes for detection can be fabricated at the same time as electrodes for driving the electrophoresis, making the overall microfabrication process very efficient [2]. In order to evaluate the efficacy of the strategies developed in the laboratory, a series of phenolic compounds

\section{Carlos D Garcia}

Department of Chemistry, The University of Texas at San Antonio, One UTSA Circle, San Antonio, TX 78249, USA

Tel. + + 2104585774

Fax: + I 2104587428

E-mail: carlos.garcía@utsa.edu 


\section{News \& Analysis I Research Spotlight}
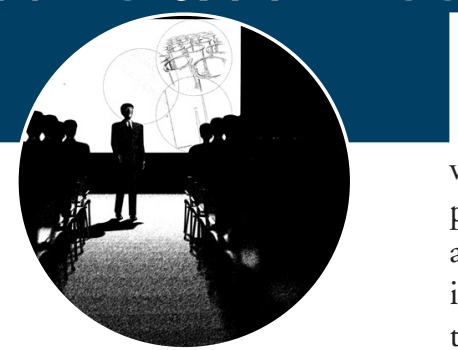

were used as model analytes. Advantageously, phenolic compounds can be ionized at moderate $\mathrm{pH}$ values $(<10)$, display strong hydrophobic interactions with capillary walls, are both electrochemically and optically active, are commercially available and are important to a variety of biological processes.

Considering the aforementioned advantages, the first priority was to demonstrate the possibility of analyzing different phenolic compounds using CE, microchips and electrochemical detection. This was initially achieved by analyzing three priority pollutants [3], as defined by the US Environmental Protection Agency.
Those studies also allowed to demonstrate that electrochemical detection can be effectively used for the analysis of phenolic compounds, avoiding electrode fouling [4-7]. Similar conditions were used to perform the analysis of other phenolic compounds, including acids [8], antioxidants [9] and pharmaceuticals [10]. It is also worth highlighting that the use of carbon-ink electrodes (FIGURE I) enabled the analysis of catecholamines down to nanomolar concentrations, without preconcentration, and using only $1.5 \mathrm{nl}$ of sample [11].

Although highly efficient separations were obtained, decreasing the analyte-wall
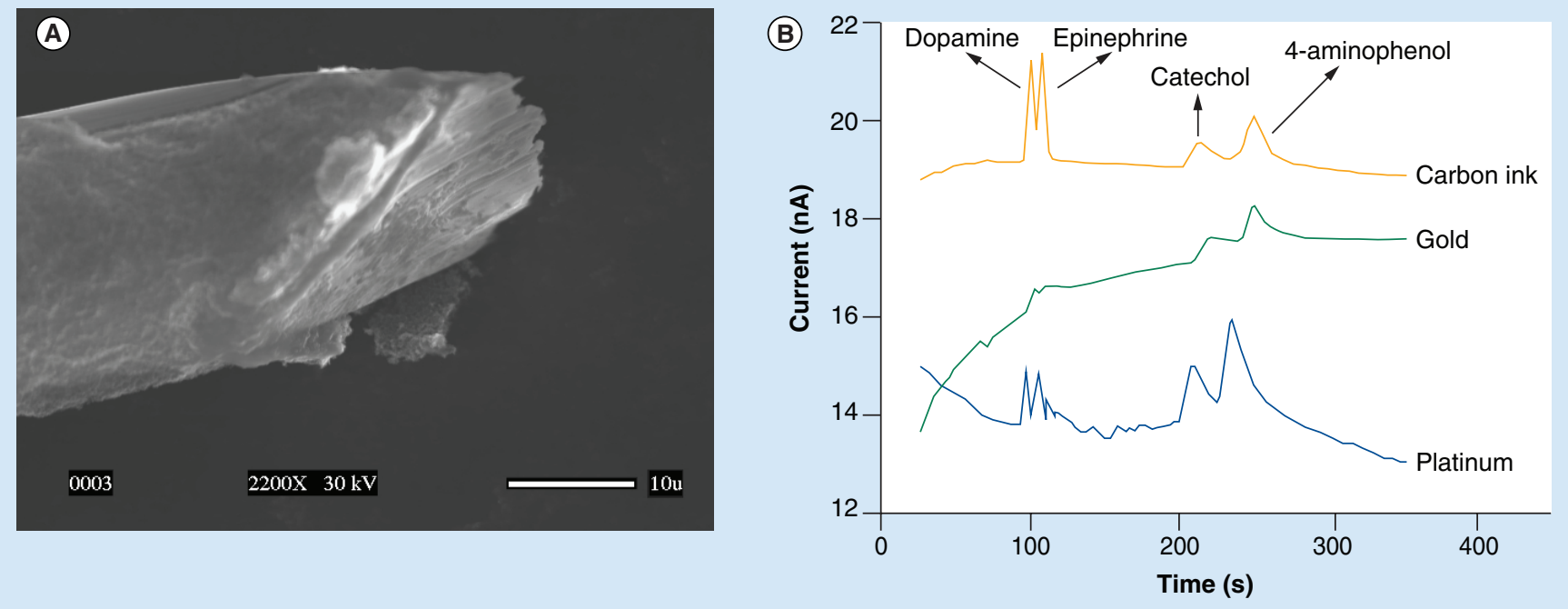

Figure 1. Integration of carbon-ink electrodes to microchip-capillary electrophoresis. (A) SEM picture of a cylindrical gold electrode coated with seven layers of carbon ink. (B) Electropherograms obtained with carbon-coated, gold and platinum electrodes for dopamine, epinephrine, catechol and 4-aminophenol. Adapted with permission from [11].

(A)

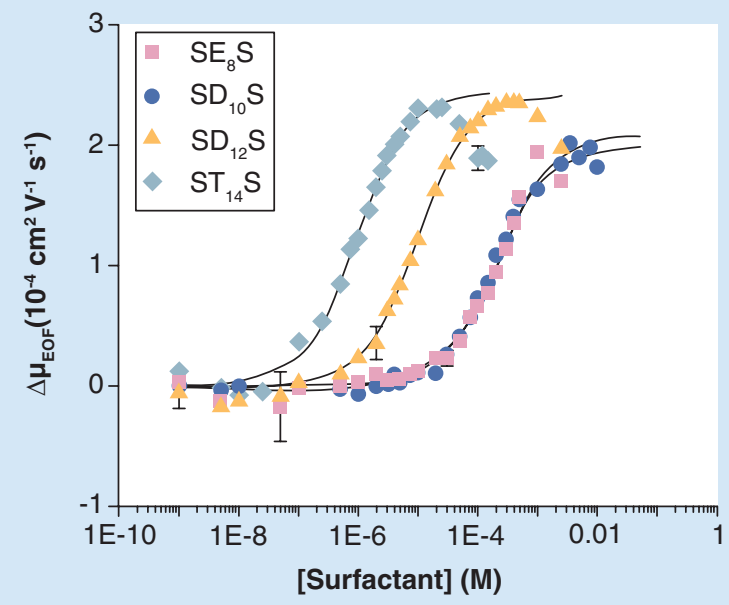

(B)

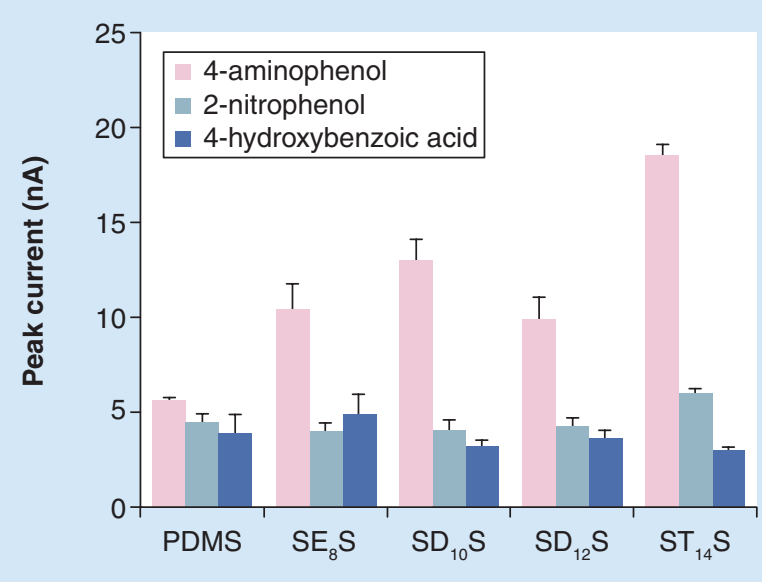

Figure 2. Effect of sodium 2-ethylhexyl sulfate, sodium decyl sulfate, sodium dodecyl sulfate and sodium tetradecyl sulfate on the $\mu \mathrm{EOF}$ and peak current. (A) $\mu$ EOF. Reproduced with permission from [13] () 2007 American Chemical Society. (B) The peak current of different phenolic compounds. Conditions described in $[13,14]$.

EOF: Electro-osmotic flow; PDMS: Poly(dimethylsiloxane); $\mathrm{SD}_{10}$ S: Sodium decyl sulfate; $\mathrm{SD}_{12}$ S: Sodium dodecyl sulfate;

$\mathrm{SE}_{8} \mathrm{~S}$ : Sodium 2-ethylhexyl sulfate; $\mathrm{ST}_{14} \mathrm{~S}$ : Sodium tetradecyl sulfate. 
interactions and enhancing the stability of the EOF was critical to further improve CE and microchip-CE separations. Considering this, our group developed a simple and inexpensive method to coat silica capillaries with montmorillonite, a natural clay [12]. In addition, we studied the adsorption of alkyl surfactants with different chain lengths and head groups to poly(dimethylsiloxane) (PDMS), one of the most commonly used materials to fabricate microchip-CE devices. Based on EOF measurements [13], the affinity constant of different surfactants was derived and related to the corresponding critical micellar concentration. Besides enabling control of the EOF, decreasing the analysis time and increasing the separation efficiency, the addition of surfactants significantly improved the performance of the electrochemical detection step (FIGURE 2) $[13,14]$.

Collaborations with other faculty members have enabled the application of microchips to the analysis of chemical warfare agents [15], the development of the first 'lab-on-a-robot' [16] and the multivariate evaluation of the separation conditions for five bisphenols [17].

\section{Interaction of proteins with nanomaterials}

With the aim to improve the selectivity of microchip-CE devices, our group investigated the possibility of integrating an immobilized enzyme reactor at the end of the separation channel [18]. Although this modification improved the selectivity of the device, the peak widths were significantly compromised. For that reason, and considering the potential advantages of carbon nanotubes (CNTs) [19], our group decided to investigate the possibility of integrating biosensors. The hypothesis of the project was that a clear understanding of the driving forces and consequences of the adsorption process would lead to a more rational way to develop CNTbased sensors. In this regard, laser reflectometry was used to perform the first kinetic study (followed in real time) of the interaction of albumin with transparent films of CNTs [20]. In order to obtain information related not only to the adsorption process but also the structure, thickness [21], optical constants and microstructure of films [22], our laboratory is now focused on the use of variable angle spectroscopic ellipsometry $[23,24]$. Variable angle spectroscopic ellipsometry enabled the possibility of extending the scope of the project to DNA [25] and other proteins, such as albumin [26], D-amino acid oxidase [27], catalase [28] and glucose oxidase [29]. In general, our results demonstrated that the activity of enzymes adsorbed to CNT is not only exclusively proportional to the amount of adsorbed protein (which is the only variable optimized in most systems; Figure 3A) but also to the initial adsorption rate (FIgure 3B). These observations are critical to develop efficient biosensors.

Besides all the well-known advantages of CNTs, our laboratory demonstrated that 3D scaffolds of CNTs can significantly increase the amount of enzymes adsorbed per unit area, preserve the catalytic activity of the adsorbed molecules and provide significant improvements $(300 \%)$ in the sensitivity of the resulting
(A)

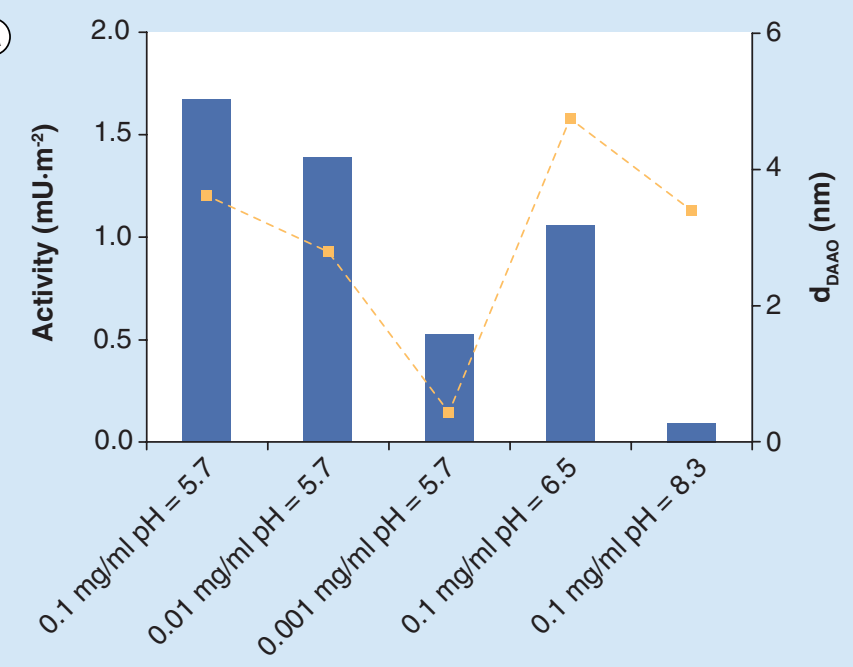

(B)

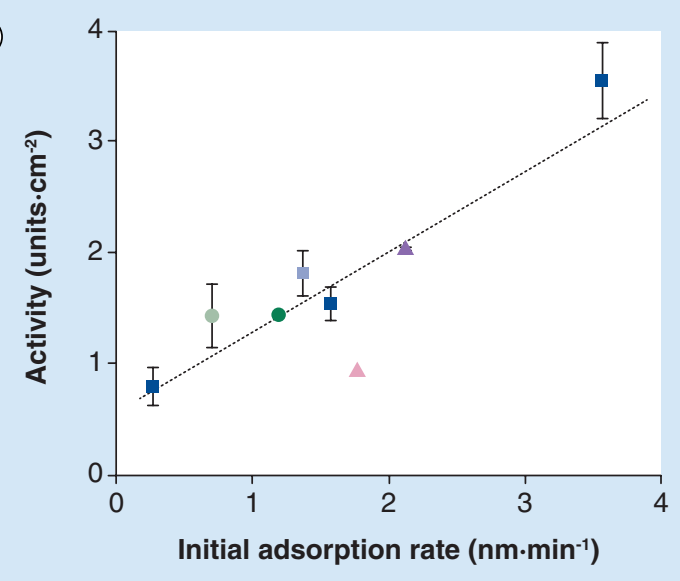

Figure 3. The enzymatic activity of D-amino acid and catalase adsorbed to carbon nanotubes. (A) Effect of experimental conditions. Reproduced with permission from [27] (C) 2009 American Chemical Society. (B) Effect of initial adsorption rate. Reproduced with permission from [28] @ 2010 American Chemical Society. Conditions as described in [27,28]. 
biosensors [29]. Furthermore, the conclusions of these studies allowed the rational selection of the experimental conditions required to develop a CNT-based biosensor for glutamate with a wide linear range $(0.01-10 \mu \mathrm{M})$, low detection limit (10 nM, $S / N \geq 3$ ), fast response time ( $\leq 5 \mathrm{~s})$ and good stability [30]. More recently, and aiming to provide rational guidelines to improve the biocompatibility of PDMS substrates, the adsorption kinetics of fibrinogen, collagen type-I and bovine serum albumin to PDMS was investigated [31]. As can be observed in Figure 4, although the three model proteins can be adsorbed to PDMS, different adsorption rates and saturation amounts were obtained indicating that specific conditions are required for the adsorption of each protein. Most importantly, these results demonstrated that the adsorption kinetics can have a major effect on the subsequent adhesion and morphology of cells.

\section{Future perspective}

Clearly, the combination of instrumentation, microfluidics, nanomaterials and proteins has the potential to provide significant improvements in the analysis of biological molecules. We will continue working in these main areas of research, aiming to provide rational guidelines to select the conditions to perform more efficient sample preparation, separation and detection steps. Because we ultimately aim to develop microfluidic devices that combine the advantages of different approaches in the laboratory, one critical aspect of our research projects is that they should be compatible with each other. In addition to these projects, we are starting to develop computational tools to understand the interactions of proteins with solid surfaces at the molecular level. We envision this approach to become one of the most efficient ways to complement the development of analytical devices. Although the current use of nanomaterials and nanostructured films in analytical chemistry is a fairly young approach that mixes art, intuition and science, many researchers around the world have recognized their utility.

\section{Acknowledgements}

The author would like to express the deepest appreciation to all the colleagues who participated in the projects described in this article.

Financial \& competing interests disclosure Financial support for these projects has been provided in part by the University of Texas at San Antonio, the National Institutes of Health through the National Institute of General Medical Sciences (1SC3GM081085, 2SC3 GM081085) and the Research Centers at Minority Institutions (G12MD007591). Financial support for an additional project, aimed at providing international training opportunities in microfluidics, has been provided by the National Science Foundation (OISE-0965814). The author has no other relevant affiliations or financial involvement with any organization or entity with a financial interest in or financial conflict with the subject matter or materials discussed in the manuscript apart from those disclosed.

No writing assistance was utilized in the production of this manuscript.
(A)

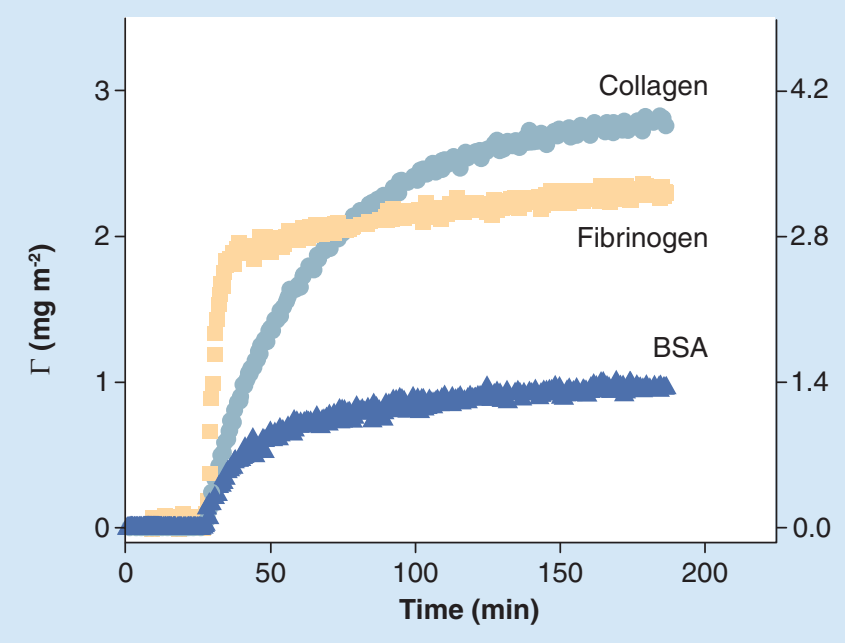

(B)

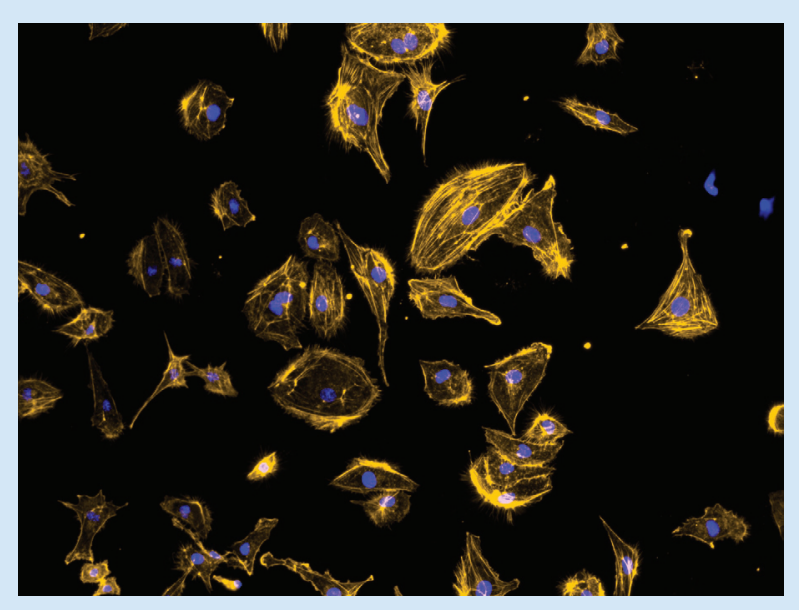

Figure 4. Interaction of endothelial cells with protein-coated poly(dimethylsiloxane). (A) Dynamic adsorption of BSA, fibrinogen and collagen onto the nanostructured poly(dimethylsiloxane) films. (B) Photograph of human endothelial cells on a substrate coated with collagen, under optimum conditions (as selected by adsorption studies). Magnification: 20x.

Adapted with permission from [31] @ 2011 Royal Society of Chemistry. 
- Research efforts in the Microanalytical Chemistry group at UTSA have focused on the development of novel analytical strategies that can be integrated to microfluidic devices.

- The final goal is to significantly improve the performance of capillary electrophoresis (CE) and microchip-CE devices, particularly when applied to the analysis of biological molecules.

- The integration of electrochemical methods has allowed nanomolar limits of detection, enabling the application of this technology to target a series of real samples.

- Complementary information obtained by variable angle spectroscopic ellipsometry has significantly improved the understanding of the driving forces and consequences of the interaction between proteins and nanomaterials.

- The implications of these results have wide applicability to the development of devices where the activity of a biorecognition element determines the overall performance.

\section{References}

1 Felhofer JL, Blanes L, Garcia CD. Recent developments in instrumentation for capillary electrophoresis and microchipcapillary electrophoresis. Electrophoresis 31, 2469-2486 (2010).

2 Garcia CD, Henry C. Coupling electrochemical detection with microchip capillary electrophoresis. In: BioMEMS: Technologies and Applications. Wang W, Soper SA (Eds). Taylor and Francis Group, Boca Raton, FL, USA, 265-298 (2007).

3 Ding Y, Garcia CD. Pulsed amperometric detection with poly(dimethylsiloxane)fabricated capillary electrophoresis microchips for the determination of EPA priority pollutants. Analyst 131(2), 208-214 (2006).

4 Garcia CD, De Pauli CP, Ortiz PI. Electrochemical characterization of glassy carbon electrodes modified by resol mixtures. J. Electroanal. Chem. 510(1-2), 115-119 (2001).

5 Garcia CD, Ortiz PI. BHA and TBHQ quantification in cosmetic samples. Electroanalysis 12(13), 1074-1076 (2000).

6 Garcia CD, Ortiz PI. Glassy carbon electrodes modified with different electropolymerized resol prepolymer mixtures for phenol and derivatives quantification. Anal. Sci. 15(5), 461-465 (1999).

7 Garcia G, Garcia CD, Ortiz PI, De Pauli CP. Reflectometry applied to electrochemically generated phenoxy radical adsorption monitoring. J. Electroanal. Chem. 519(1-2), 53-59 (2002).

8 Ding Y, Garcia CD. Application of microchip - capillary electrophoresis to follow the degradation of phenolic acids by aquatic plants. Electrophoresis 27(24), 5119-5127 (2006).

9 Ding Y, Mora MF, Garcia CD. Analysis of alkyl gallates and nordihydroguaiaretic acid using plastic capillary electrophoresis microchips. Anal. Chim. Acta 561(1-2), 126-132 (2006).
10 Ding Y, Garcia CD. Determination of non-steroidal anti-inflammatory drugs in serum by capillary electrophoresis microchip and electrochemical detection. Electroanalysis 18(22), 2202-2209 (2006).

11 Ding Y, Ayon A, Garcia CD. Electrochemical detection of phenolic compounds using cylindrical carbon-ink electrodes and microchip capillary electrophoresis Anal. Chim. Acta 584(2), 244-251 (2007).

12 Mora MF, Garcia CD. Electrophoretic separation of phenolic contaminants using fused silica capillaries coated with montmorillonite. Electrophoresis 28(8), 1197-1203 (2007).

13 Mora MF, Giacomelli CE, Garcia CD. Electrophoretic effects of the adsorption of anionic surfactants to poly(dimethylsiloxane) - coated capillaries. Anal. Chem. 79, 6675-6681 (2007).

14 Ding Y, Mora MF, Merrill GN, Garcia CD. The effects of alkyl sulfates on the analysis of phenolic compounds by microchip capillary electrophoresis with pulsed amperometric detection. Analyst 132(10), 997-1004 (2007).

15 Ding Y, Rogers K, Garcia CD. Poly(dimethylsiloxane) microchip electrophoresis with contactless conductivity detection for measurement of chemical warfare agent degradation products. Anal. Lett. 41(2), 335-350 (2008).

16 Berg C, Valdez DC, Bergeron P, Mora MF, Garcia CD, Ayon A. Lab-on-a-robot: integrated microchip - capillary electrophoresis, power supply, potentiostat, wireless unit, and controller circuitry. Electrophoresis (29), 4914-4921 (2008).

17 Felhofer J, Hanrahan G, Garcia CD. Multivariate versus univariate optimization of separation conditions in micellar electrokinetic chromatography. Talanta 77(3), 1172-1178 (2008).
18 Blanes L, Mora Maria F, do Lago Claudimir L, Ayon A, Garcia CD. Lab-on-achip biosensor for glucose based on a packed immobilized enzyme reactor. Electroanalysis 19(23), 2451-2456 (2007).

19 Scida K, Stege PW, Haby G, Messina GA, Garcia CD. Recent applications of carbonbased nanomaterials to analytical chemistry: a critical review. Anal. Chim. Acta 691, 6-17 (2011).

20 Valenti LE, Fiorito PA, Garcia CD, Giacomelli CE. The adsorption-desorption process of bovine serum albumin on carbon nanotubes. J. Colloid Interface Sci. 307(2), 349-356 (2007).

21 Nejadnik MR, Garcia CD. Staining proteins: a simple method to increase the sensitivity of ellipsometric measurements in adsorption studies. Colloids Surf. B 82(1), 253-257 (2011).

22 Soetedjo H, Mora MF, Garcia CD. Optical properties of single-wall carbon nanotube films deposited on $\mathrm{Si} / \mathrm{SiO}_{2}$ wafers. Thin Solid Films 518(14), 3954-3959 (2010).

23 Mora MF, Valenti LE, Garcia CD, Giacomelli CE. Driving forces and consequences of the adsorption of proteins to carbon nanotubes. In: Advanced Bioceramics in Nanomedicine and Tissue Engineering. Trans Tech Publications, Zurich, Switzerland, 75-94 (2010).

24 Mora MF, Wehmeyer J, Synowicki R, Garcia CD. Investigating the adsorption of proteins via spectroscopic ellipsometry. In: Biological Interactions on Material Surfaces: Understanding and Controlling Protein, Cell, and Tissue Responses. Bizios R, Puleo D (Eds.). Springer, NY, USA (2009).

25 Carot ML, Torresi RM, Garcia CD, Esplandiu MJ, Giacomelli CE. Electrostatic and hydrophobic interactions involved in CNT biofunctionalization with short ssDNA. J. Phys. Chem. C 114(10), 4459-4465 (2010). 


\section{News \& Analysis I Research Spotlight}

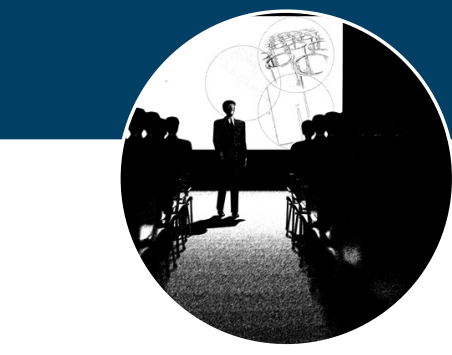

26 Wehmeyer J, Bizios R, Garcia CD. Adsorption of bovine serum albumin to nanostructured thin-films of $\mathrm{TiO}_{2}$. Mat. Sci. Eng. C 30 (2), 277-282 (2010).

27 Mora MF, Giacomelli CE, Garcia CD. Interaction of D-amino acid oxidase to carbon nanotubes: implications in the design of biosensors. Anal. Chem. 81, 1016-1022 (2009).

28 Felhofer JL, Caranto J, Garcia CD. Adsorption kinetics of catalase to thin films of carbon nanotubes. Langmuir 26, 17178-17183 (2010).
29 Nejadnik MR, Garcia CD. Nanoscale scaffolds of carbon nanotubes for immobilization of glucose oxidase. Electroanalysis 23, 1462-1469 (2011).

30 Khan R, Gorski W, Garcia CD. Nanomolar detection of L-glutamate using screen-printed electrodes containing multi-wall carbon nanotubes. Electroanalysis 23, 2357-2363 (2011).

31 Chumbimuni-Torres KY, Coronado RE, Mfuh AM et al. Adsorption of proteins to thin-films of PDMS and its effect on the adhesion of human endothelial cells. RSC Advances (4), 706-714 (2011). 